\title{
Reserva cognitiva y déficit en la planificación en pacientes con esclerosis múltiple.
}

Cognitive reserve and planning deficit in patients with multiple sclerosis.

\begin{abstract}
Lilia Mestas a ${ }^{\text {, Judith Salvador }}{ }^{\text {b }}$, Fernando Gordillo ${ }^{\text {c. }}$
${ }^{a}$ Facultad de Estudios Superiores Zaragoza (FESZ). División de Investigación y Posgrado. Residencia en Neuropsicología Clínica. Universidad Nacional Autónoma de México (UNAM), ${ }^{b}$ Facultad de Estudios Superiores Zaragoza (FESZ). División de Investigación y Posgrado. Residencia en Neuropsicología Clínica. Universidad Nacional Autónoma de México (UNAM), ${ }^{c}$ Facultad de Psicología. Departamento de Psicología Básica, Psicobiología y Metodología.Universidad de Salamanca, España.
\end{abstract}

Correspondencia: Lilia Mestas (lilia_mestas@yahoo.com.mx)

Recibido: 01/06/2011; aceptado: 17/09/2011

RESUMEN: La esclerosis múltiple afecta el sistema nervioso produciendo alteraciones cognitivas. La memoria es uno de los más afectados, no obstante la naturaleza ó factor primario de alteración aún no se clarifica. Objetivo: Estudiar las diferencias en el procesamiento de la información verbal en un grupo de pacientes con esta patología neurológica respecto a un grupo de control sano. Para ello se analizó el desempeño neuropsicológico de 16 pacientes con esclerosis múltiple de tipo remitente-recurrente y un grupo de control equiparado en las variables sociodemográficas. A los dos grupos se les aplicó el Test de Aprendizaje Verbal España-Complutense, y se realizó una comparación entre diversos índices de la prueba, además de un análisis de correlaciones entre las variables sociodemográficas del grupo experimental para identificar algún factor protector. Resultados: el grupo de esclerosis múltiple obtuvo rendimientos inferiores en los índices analizados respecto al de control, además de una correlación positiva entre años de escolaridad y el inicio de la enfermedad y entre el inicio de la enfermedad y la ocupación. Los datos encontrados parecen indicar que los pacientes con esclerosis múltiple tienen poca capacidad de planificación, que incide de manera directa en su desempeño en tareas de memoria y aprendizaje.

PALABRAS CLAVE: funciones ejecutivas, aprendizaje, procesamiento mnésico, información verbal.
ABSTRACT: Multiple sclerosis affects the nervous system causing cognitive impairment. Memory is one of the most affected, notwithstanding the nature or primary factor of disturbance is not yet clarified. Objective: To study the differences in verbal information processing in a group of this neurological pathology patients compared to healthy control group. To do this we examined the neuropsychological performance of 16 patients with multiple sclerosis relapsing-remitting type and a control group. The two groups were administered the Verbal Learning Test SpainComplutense and a comparison between various test indices in both groups, and an analysis of correlation between socio-demographic variables in the experimental group to identify a protective factor. Results: Multiple sclerosis group obtained lower scores on indices analyzed with respect to control, as well as positive correlation between years of schooling and the onset of the disease and between the onset of the disease and occupation. The data found suggest that multiple sclerosis patients have little ability to plan, which directly affects their performance in learning and memory tasks.

KEY WORDS: Executive functions, learning, mnesic processing, verbal information.

\section{Introducción}

La esclerosis múltiple (EM) afecta al sistema nervioso a través de una progresiva desmielinización (1). Se ha establecido como la enfermedad neurológica más frecuente en adultos jóvenes de Europa y América, y una de las principales causas de invalidez para este grupo poblacional (2). Diferentes factores parecen 
predisponer a contraer la enfermedad aunque también se mencionan algunos agentes protectores, como el nivel de educación $(3,4)$. Sin embargo, hasta la fecha la etiología precisa de esta enfermedad es desconocida (5), por lo que resulta necesario su abordaje desde diferentes perspectivas con el fin de situarla en un rango de manejo clínico adecuado que permita mejorar la calidad de vida del paciente.

Las dificultades mnésicas más documentadas en estos enfermos son las referidas a la memoria de trabajo y episódica (6-10). La mayoría de estos trabajos coinciden en afirmar que las dificultades en la recuperación de la información serían la causa primaria de los trastornos de memoria, habiendo poca influencia del registro y la codificación de los datos, aunque otros enfoques participan en la idea de que los problemas en la codificación repercuten de manera directa y primaria en los déficit de memoria observados $(11,12)$. Algunos indican que en la base de las dificultades observadas en la memoria episódica se encuentra una alteración de la memoria de trabajo, al impedirse la correcta funcionalidad ejecutiva que permite mantener la información relevante en presencia de la irrelevante (13-16). Se piensa por tanto, que los déficit encontrados en la memoria episódica y el aprendizaje podrían estar condicionados por dificultades en las funciones perceptuales, el lento procesamiento de la información, y una alta susceptibilidad a la interferencia o compromiso en las funciones ejecutivas (FE).

En este sentido, el uso e implementación de los procesos y estrategias de memoria, al ser controlados, requieren una alta demanda de las FE, por lo que el funcionamiento adecuado de procesos ejecutivos como la organización y planificación, la monitorización y la flexibilidad cognitiva, ejercen un papel fundamental para la elaboración de estrategias de memoria eficaces para incrementar su capacidad (12). La planificación es uno de los aspectos más vinculados al recuerdo (visto como una solución de problemas) ya que determina el modo de abordar una tarea de forma que su desempeño sea óptimo. Este proceso se ha definido como la capacidad de formar estrategias, coordinar, secuenciar la actividad mental y mantener esa información online. Su alteración podría originar afectaciones a diferentes niveles (17), y específicamente, en el ámbito de la memoria y el aprendizaje.

En contextos clínicos, diversas pruebas se han usado para la valoración de la memoria y el aprendizaje en pacientes con EM. Entre las más utilizadas se encuentra el California Verbal Learning Test (CVLT) para poblaciones de habla inglesa (7) y el Test de Aprendizaje Verbal España-Complutense (TAVEC) en lengua española. Este test permite evaluar la memoria episódica verbal y el aprendizaje de palabras a través de diferentes índices que delimitan los procesos de recuperación y codificación. Al mismo tiempo, introduce listas de interferencia que permiten estudiar las dificultades en la capacidad de retener información relevante en presencia de información distractora, por lo tanto, se considera un paradigma válido para profundizar en las causas que determinarían los déficit mnésicos observados en pacientes con 
ORIGINALES Y REVISIONES

algún déficit de memoria, específicamente, en pacientes con EM, ya que cuantifica, describe y analiza diferentes estructuras de la memoria. Sin embargo, la dificultad central radica en determinar qué origina que un paciente presente tales problemas.

En este sentido, algunos autores han explorado la posible relación entre algunas variables como el grado de discapacidad puntuado en la Kutzke Expanded Disability Status Scale (EDDS) y los años de evolución de la enfermedad, encontrando que son pobres predictores para el deterioro cognitivo (18), por lo que el interés ahora se focaliza en determinar factores medulares que expliquen o condicionen la expresión de déficit cognitivos, tales como los años de escolaridad, la ocupación, los años de inicio de la enfermedad, etc. Desde esta perspectiva se puede señalar que la escolarización proporciona a las personas las herramientas para poder decidir entre los diferentes caminos alternativos o soluciones, así por ejemplo, brinda opciones para implementar planes a seguir y en caso de no ser adecuados, nos capacita para flexibilizar las estrategias (aspectos vinculados a las funciones ejecutivas) (19), lo que podría "proteger" en casos como la EM.

Dentro de este marco teórico, el propósito de la presente investigación se centrará en estudiar las diferencias en el procesamiento de la información verbal en un grupo de personas con EM respecto a un grupo control sano, analizando el desempeño de éstos en los diferentes índices de una tarea de aprendizaje de palabras, y haciendo énfasis en aquellos aspectos que se relacionen con dificultades en la planificación de la tarea pues se hipotetiza que el rendimiento de los pacientes con EM estará justificado por dificultades en este proceso. Adicionalmente, se realizará un análisis de correlación entre las características socio-demográficas de la muestra estudiada para conocer si se puede apreciar algún "factor protector" en estos pacientes, ya que estudios previos han señalado la existencia de éstos (3) y pondría de manifiesto no sólo las bases anatomofisiológicas de la enfermedad también su conjugación con factores sociales (enfoque ecológico).

\section{Sujetos y Método}

\section{Participantes}

El grupo de trabajo se compuso de 16 sujetos con diagnóstico de EM tipo remitente-recurrente, clínicamente confirmado por el neurólogo a cargo basándose en los criterios de Poser et al. (20), y 16 sujetos sanos que participaron como controles pareados en edad, escolaridad y ocupación con los pacientes (Tabla 1). Todos aceptaron participar de manera voluntaria en el estudio. Fueron excluidos de la muestra los sujetos con antecedentes de drogadicción, alcoholismo, trastornos neurológicos, psiquiátricos o psicológicos. 
Tabla 1

Media y desviación estándar de los datos demográficos y clínicos de los 32 sujetos participantes en el estudio.

\begin{tabular}{|ll|l|l|}
\hline & & Esclerosis Múltiple & Controles sanos \\
\hline Casos & & 16 & 16 \\
\hline Edad (años) & Femenino & $\mathrm{M}=37,94(\mathrm{DT}=8,95)$ & $\mathrm{M}=37,81(\mathrm{DT}=9,03)$ \\
Sexo & Masculino & $50 \%$ & $50 \%$ \\
& & $50 \%$ & $50 \%$ \\
Escolaridad (años) & & $\mathrm{M}=16,62(\mathrm{DT}=4,20)$ & $\mathrm{M}=16,69(\mathrm{DT}=4,12)$ \\
Evolución (años) & & $\mathrm{M}=5,25(\mathrm{DT}=4,53)$ & - \\
Edad de inicio & & $\mathrm{M}=32,69(\mathrm{DT}=8,25)$ & - \\
Ocupación & Profesionista & $56,25 \%$ & $56,25 \%$ \\
& Administrativo & $31,25 \%$ & $31,25 \%$ \\
& Desempleado & $12,50 \%$ & $12,50 \%$ \\
\hline
\end{tabular}

\section{Instrumentos}

Se aplicó a todos los sujetos el Mini-Examen del Estado Mental (MMSE) (21), que es un test breve de valoración del estado cognitivo, y se constató que ninguno de los participantes mostraba deterioro. Para la evaluación de la memoria episódica se utilizó el Test de Aprendizaje Verbal España-Complutense (TAVEC) (30). Esta prueba consta de tres listas de palabras que se presentan como "listas de compras": a) de aprendizaje (lista A), b) de interferencia (lista B), y c) de reconocimiento. En el análisis del TAVEC se empleó el primer nivel de interpretación de los datos que corresponde a la cuantificación del funcionamiento del sistema de memoria de los sujetos que sugieren Benedet y Alejandre (22). Esta cuantificación del funcionamiento del sistema de memoria se divide en dos partes, cada una de ellas a su vez se subdivide en apartados específicos. La primera parte abarca las pruebas de recuerdo mientras que la segunda involucra la prueba de reconocimiento.

Como medida de la memoria episódica verbal se utilizaron los siguientes índices extraídos del TAVEC:

Valoración de la capacidad de aprendizaje: Se tomaron en cuenta el número de palabras correctamente recordadas en el ensayo 1 (E_1_Lista A) y 5 (E_5_Lista A), así como el sumatorio de los cinco ensayos (E_T_Lista A). Con estas medidas se pretende comprobar la progresión en el aprendizaje del sujeto desde el ensayo 1 al 5.

Valoración por tipo de memoria. Se midieron diferentes tipos de memoria, 1) recuerdo libre a corto plazo (RLCP); 2) recuerdo libre a largo plazo (RLLP) y 3) reconocimiento.

Valoración de las funciones ejecutivas $(F E)$. Se utilizaron las intrusiones y perseveraciones en el mismo sentido de identificación con las FE que el utilizado 
ORIGINALES Y REVISIONES

en el trabajo de Higueras, Jiménez-Martín, Muñoz, Mateo y de Andrés (23). En las intrusiones se registra el número de palabras que pertenecen a la lista de interferencia, y que el sujeto indica erróneamente como pertenecientes a la lista A. Un elevado número de intrusiones podría estar reflejando una menor capacidad de inhibición del lóbulo frontal (24). Para las perseveraciones se registró el número de palabras que el sujeto repite al intentar recordar, y que pertenecen a la lista A. Ambas medidas se contabilizan desde el ensayo 1 y durante toda la prueba.

Valoración de los procesos de codificación. Se utilizaron los índices de estrategias semánticas (E_Semántica Lista A) y seriales (E_Seriales Lista A) de la lista A. El primero indica la frecuencia con la que el sujeto nombra una palabra correcta de una categoría semántica de manera inmediata a otra palabra de la misma categoría. Por otro lado, el índice de estrategias seriales indica la frecuencia con la que se nombran palabras en el mismo orden en el que aparecieron en la lista de aprendizaje. También se utilizaron el recuerdo libre con claves a corto (Claves_CP) y largo plazo (Claves_LP), que nos permitió inferir si los problemas derivan de la codificación o la recuperación.

Valoración de los procesos de recuperación. Se utilizaron los índices de estrategias seriales y semánticas a corto y largo plazo (E_Semántica RLCP; E_Semántica RLLP; E_Serial RLCP; E_Serial RLLP), que nos permitió comprobar el grado de utilización de éstas a lo largo de toda la prueba.

\section{Resultados}

Para el análisis de los índices obtenidos en el TAVEC se realizó una $t$ de Student, en la que comparamos los resultados obtenidos en dichos índices (puntuación normalizada), entre el grupo de sujeto con EM y el grupo de sujeto control sanos. En la tabla 2 se muestran los resultados de dicho análisis, así como la media (M) y la desviación típica (DT) de los dos grupos.

El grupo de pacientes con EM difiere en sus medias respecto al grupo de controles sanos en todos los tipos de memoria registrados, tanto en recuerdo libre, a corto (RLCP; $\mathrm{t}(2,599), \mathrm{p}=.014)$ y largo plazo (RLLP; $\mathrm{t}(4,558), \mathrm{p}=.000)$, como en memoria de reconocimiento $(\mathrm{t}(2,677), \mathrm{p}=.012)$. Las diferencias evidencian un peor rendimiento de los sujetos con EM en todos estos índices (tabla 2).

Este resultado es congruente con los datos obtenidos para el grado de aprendizaje, medido a través del número de aciertos en recuerdo inmediato en los cinco ensayos consecutivos de la primera parte de la prueba. No se muestran diferencias en el primer ensayo (E_1 Lista $\mathrm{A} ; \mathrm{t}(1,804), \mathrm{p}=.081)$, pero sí en el quinto (E_5 Lista $A ; t(4,435), p=.000)$ y en el sumatorio de los cinco ensayos ( $E_{-} T$ Lista $A ; t(3,972)$, 
$\mathrm{p}=.001)$. Este resultado nos informa de que, si bien tras el primer ensayo no se dan diferencias, en el transcurso de los cinco ensayos éstas empiezan a manifestarse como resultado de los déficit en la capacidad de aprender de los ensayos anteriores.

Para conocer en qué aspectos de la memoria se focaliza este déficit, se analizaron los resultados obtenidos en los procesos de recuperación y codificación. Como puede verse en la tabla 2, los sujeto con EM tienen dificultades para utilizar las estrategias semánticas, y esto se refleja en las diferencias registradas respecto al grupo de control, en los índices de recuperación (E_Semántica RLCP: t(-3,557), $\mathrm{p}=.001$; E_Semántica RLCP: $\mathrm{t}(-3,362), \mathrm{p}=.002)$ y codificación (Claves_RLCP: $\mathrm{t}(-3,557), \mathrm{p}=.001$; Claves_RLLP: $\mathrm{t}(-3,362), \mathrm{p}=.002)$. Estos datos informan de que los sujetos con EM utilizan en menor grado las estrategias semánticas, y no se benefician de éstas durante la recuperación. Por último, no se encontraron diferencias en el número de intrusiones y perseveraciones entre ambos grupos, por lo que el problema de memoria podría no estar relacionado con déficit en las funciones ejecutivas.

Por último, el análisis de correlaciones bivariadas realizado sobre las variables demográficas nos informó de una correlación positiva entre la edad de inicio y los años de escolaridad $(r=.597, \mathrm{p}<0,05)$ y en el mismo sentido entre la edad de inicio y la ocupación $(\mathrm{r}=-.667, \mathrm{p}<0,01)$.

\section{Discusión y Conclusiones}

Las dificultades en la memoria y en el aprendizaje son mencionadas de manera consistente como elementos centrales en la EM (11). Sin embargo, aún no queda clara la naturaleza de los déficit antes mencionados. El propósito de esta investigación fue abordar las diferencias en el procesamiento de la información verbal en un grupo de pacientes con EM respecto a un grupo control sano, examinándose el desempeño de éstos en diferentes índices, además de analizar la relación entre las características socio-demográficas de la muestra estudiada.

En referencia al desempeño cognitivo, al realizar el análisis del procesamiento de información verbal tanto en pacientes con EM y en el grupo control, se obtuvieron diferencias en todos los índices estudiados. Sin embargo, el hecho de haber registrado problemas de manera general en cuanto a las diferentes estructuras de la memoria (a corto y a largo plazo), el reconocimiento y la modalidad del recuerdo (libre y con claves), señala que los pacientes con EM tienen un defecto inicial en la codificación de la información, lo que afecta de manera secundaria a todo el proceso, es decir, que hay un elemento primario que condiciona los resultados obtenidos 
Tabla 2

Resultados de la t de Student para el grupo de esclerosis y el control.

\begin{tabular}{|c|c|c|c|c|c|c|}
\hline MEDIDA & ÍNDICE & $\mathbf{t}$ & Sig. & Gl & $\begin{array}{c}\text { Controles sanos } \\
\text { M (DT) }\end{array}$ & $\begin{array}{c}\text { Esclerosis } \\
\text { M(DT) }\end{array}$ \\
\hline \multirow[t]{3}{*}{ APRENDIZAJE } & E_1 (Lista A) & 1,804 & .081 & 30 & $0,13(1,36)$ & $-0,63(0,96)$ \\
\hline & E_5 (Lista A) & 4,435 & .000 & 30 & $0,44(0,81)$ & $-1,31(1,35)$ \\
\hline & E_T (Lista A) & 3,972 & .001 & 22,94 & $0,25(0,68)$ & $-1,19(1,28)$ \\
\hline \multirow[t]{4}{*}{ RECUPERACIÓN } & $\begin{array}{c}\text { E_Semántica } \\
\text { RLCP }\end{array}$ & $-3,703$ & .001 & 22,06 & $-1,06(0,57)$ & $0,13(1,15)$ \\
\hline & $\begin{array}{l}\text { E_Semántica } \\
\text { RLLP }\end{array}$ & $-3,046$ & .005 & 30 & $-1,06(0,77)$ & $-0,06(1,06)$ \\
\hline & $\begin{array}{l}\text { E_Seriales } \\
\text { RLCP }\end{array}$ & 4,86 & .630 & 30 & $0,06(0,85)$ & $-0,06(0,57)$ \\
\hline & $\begin{array}{c}\text { E_Seriales } \\
\text { RLLP }\end{array}$ & 4,88 & .629 & 30 & $0,00(0,82)$ & $-0,13(0,62)$ \\
\hline \multirow[t]{4}{*}{ CODIFICACIÓN } & \begin{tabular}{|l|} 
Claves_RLCP \\
\end{tabular} & $-3,557$ & .001 & 30 & $-1,31(1,19)$ & $0,13(1,09)$ \\
\hline & Claves_RLLP & $-3,362$ & .002 & 30 & $-0,94(1,12)$ & $0,25(0,85)$ \\
\hline & $\begin{array}{c}\text { E_Semántica } \\
\text { Lista A }\end{array}$ & $-1,777$ & .086 & 30 & $-0,94(0,85)$ & $-0,25(1,29)$ \\
\hline & $\begin{array}{c}\text { E_Seriales } \\
\text { Lista A }\end{array}$ & 0,275 & .786 & 30 & $-0,25(0,58)$ & $-0,70(0,62)$ \\
\hline \multirow[t]{3}{*}{ TIPO_MEMORIA } & RLCP & 2,599 & .014 & 30 & $0,13(1,02)$ & $-1,13(1,63)$ \\
\hline & RLLP & 4,558 & .000 & 30 & $0,44(0,89)$ & $-1,31(1,25)$ \\
\hline & Reconocimiento & 2,677 & .012 & 30 & $0,38(1,02)$ & $-0,63(1,09)$ \\
\hline FUNCIONES & Intrusiones & $-0,131$ & .896 & 30 & $0,25(1,29)$ & $0,31(1,40)$ \\
\hline EJECUTIVAS & Perseveraciones & $-1,106$ & .278 & 27,02 & $0,94(1,43)$ & $1,63(2,03)$ \\
\hline
\end{tabular}

En: ensayo $n(1,2,3,4,5)$.

$E_{-}^{-T}$ : suma de los aciertos em los 5 ensayos

E- Semántica: estrategia semántica

E- Serial: estrategia serial

$R \bar{L} C P$ : recuerdo libra a corto plazo

RLLA: recuerdo libre a largo plazo

Claves_RLCP: recuerdo con claves a corto plazo.

Claves $R L L P$ : recuerdo con claves a largo plazo.

* Significación estadísica de las variables, $\alpha=0.05$.

por estas personas. En este sentido, el perfil de memoria visto en la enfermedad y que se relacionaba con un perfil de demencia subcortical (reconocimiento, codificación y almacenamiento preservados) es cuestionable, ya que hay variabilidad en los resultados. Sin embargo, estas dificultades de memoria podrían ser el resultado de una alteración en otras funciones, por ejemplo, las ejecutivas (25).

En este sentido, al verificar que los participantes con EM usaban de menor manera las estrategias semánticas indica posiblemente una dificultad para estable- 
cer un plan de trabajo previo tanto para el aprendizaje como para la recuperación de los datos, lo que señala dificultades en la planificación de la actividad a realizar. No obstante, el hecho de no haber presentado intrusiones y perseveraciones de manera significativamente distinta a los controles, señalaría una capacidad conservada de organización del almacén semántico y una adecuada posibilidad de inhibición. Estos datos en conjunto apoyan la idea de que las funciones ejecutivas no son un constructo unitario sino que están integradas de diversos elementos que podrían verse afectados de manera diferencial por una enfermedad como la EM.

García-Molina et al. (26) señalaron que las funciones ejecutivas hacen referencia a una constelación de funciones cognitivas necesarias para poder enfrentar situaciones novedosas, imprevistas o cambiantes de manera adaptativa donde el sujeto diseña diferentes soluciones partiendo del conocimiento almacenado, de las demandas del medio ambiente y de las metas y objetivos perseguidos. En este sentido, para desempeñarse adecuadamente en el TAVEC son necesarios tres pasos: a) descubrir de qué manera está estructurada la prueba, b) establecer una estrategia de aprendizaje, e c) implementarla y usarla de manera consistente durante toda la prueba, lo que hace alusión al funcionamiento ejecutivo y específicamente a una adecuada planificación de la tarea, quedando definida como una memoria de tipo estratégica. Los resultados de esta investigación indican que los pacientes con EM están lejos de realizar estos pasos, sobre todo los dos últimos.

Planificar implica identificar y organizar los pasos y elementos necesarios para lograr un objetivo, e incluye la capacidad de adoptar una actitud abstracta, valorar las diferentes posibilidades y desarrollar un marco conceptual que permita dirigir la actividad (27). Las personas con EM son poco eficaces y cometen más errores en tareas que precisan de planificación, mostrando dificultades en la regulación de su conducta, y de igual manera, suelen mostrar dificultades en la toma de decisiones (28). Por lo tanto, los hallazgos obtenidos en la actual investigación muestran que la principal dificultad de los pacientes con EM se ubica no en el registro, almacenamiento o recuperación de la información, sino en un paso previo, en el establecimiento y uso adecuado y eficiente de una estrategia de aprendizaje, es decir, una alteración en la planificación.

Respecto al segundo objetivo planteado, los hallazgos de esta investigación señalan, por una parte, una correlación positiva entre el nivel de escolaridad y el inicio de la enfermedad, y por otro, el inicio de la enfermedad y la ocupación. Esto parece indicar en primer lugar, que la educación es un factor que protege y retrasa el inicio de la EM (lo que se ha denominado como factor protector) y en segundo término, que la naturaleza de la ocupación determina en cierta medida la manifestación de los síntomas, es decir, que éstos se manifiesten de manera temprana o que sean frenados. 
ORIGINALES Y REVISIONES

En este sentido, el concepto de reserva cognitiva puede ser usado para explicar estos datos. Esta noción hace referencia a la influencia que ejercen las experiencias tales como los años de escolaridad y el tipo de ocupación en el desempeño cognitivo de un individuo. La reserva cognitiva puede definirse como la capacidad de potenciar el rendimiento cognitivo a través del reclutamiento o la incorporación a diferentes redes neuronales, lo que se reflejaría clínicamente en la utilización de estrategias cognitivas alternativas. Así, los sujetos con mayor reserva procesarían de manera más eficaz que aquellos con una reserva menor, es decir, si un individuo tiene mayor reserva cognitiva tendría una mayor capacidad de apelar a redes neuronales alternativas o diferentes estrategias cognitivas en respuesta a tareas que demanden recursos crecientes (29).

En sintonía con estos datos, Sartori y Edan (30) señalaron que tener más de 40 años de edad y menos de 11 años de escolarización es un factor de riesgo muy importante para presentar defectos cognitivos en pacientes con EM. En su estudio indicaron que el $79 \%$ de los pacientes que mostraron deterioro cognitivo sólo recibieron 9 años de educación formal. Las correlaciones obtenidas en nuestro trabajo entre la edad de inicio de la enfermedad y la escolaridad y ocupación de los sujetos parecen estar en consonancia con los de Sartori y Edan (26), al tiempo que enfatizan el papel de las variables sociales y emocionales en los procesos patológicos. Sin embargo, con referencia al grado de escolarización, hay que tomar estos datos con precaución ya que podría suceder que no haya una correspondencia única entre el desempeño cognitivo y el nivel educativo, sino el cruce de diversos factores como la ocupación y las actividades realizadas de manera cotidiana por los enfermos. Estas últimas variables podrían estar englobando una mayor demanda cognitiva, por lo tanto, un reforzamiento de las redes neuronales, lo que podría conseguirse sin necesidad de un alto nivel de educación.

Finalmente si bien los resultados obtenidos no son definitivos debido al tamaño de muestra evaluada, sí indican que los pacientes con EM presentan una dificultad primaria en el proceso de planificación de la tarea, lo que incide de manera directa en la codificación inicial de la información y que repercute a su vez en los restantes procesos de memoria. Aunque el análisis de los datos del TAVEC se hizo de manera cuantitativa, el punto de vista neuropsicológico puso de manifiesto las características funcionales del desempeño de los participantes y mostró la necesidad de analizar cualitativamente el desempeño de la muestra. En este sentido, el enfoque neuropsicológico proporcionaría riqueza a las cifras obtenidas, por lo tanto, es deseable enfocarse al nivel de descripción y explicación del desempeño en tareas mnésicas y evaluar con una prueba específica de planificación, el rendimiento de estos pacientes para cruzar estos resultados con índices o tareas de memoria, además de analizar el progreso de la enfermedad junto con cada una de las funciones ejecutivas, desde volición hasta la respuesta efectiva. 


\section{BIBLIOGRAFÍA:}

(1) Anaya J, Gómez L. Bases moleculares de las enfermedades autoinmunes del sistema nervioso. En J, Anaya Y, Shoenfeld P, Correa M, García-Carrasco R, Cervera R, eds. Autoinmunidad y enfermedad autoinmune. Medellín: Corporación para Investigaciones Biológicas; 2005. p 363-372.

(2) Terré-Boliart R, Orient-López F. Tratamiento rehabilitador en la esclerosis múltiple. Rev Neurol 2007; 44: 426-31.

(3) Ghadirian P, Dadgostar B, Azani R, Maisonneuve P. A case-control study of the association between socio-demographic, lifestyle and medical history factors and multiple sclerosis. Canadian J Public Health 2001; 92: 281-5.

(4) Zorzon M, Zivadinov R, Nasuelli D, Dolfini P, Bosco A, Bratina A, et al. Risk factors of multiple sclerosis: a case-control study. Neurol Sci 2003; 24: 242-7.

(5) Mehta BK.. New hypotheses on sunlight and the geographic variability of multiple sclerosis prevalence. J Neurol Sci 2010; 292: 5-10.

(6) Bobholz J, Rao S. (2003). Cognitive dysfunction in multiple sclerosis: a review of recent developments. Curr opin neurol 2003; 16: 283-8.

(7) Defer G., Daniel F. Marié R. Study of episodic memory in multiple sclerosis using the California Verbal Learning Test: the data favour altered encoding. Rev Neurol 2006; 162: 852-7.

(8) Lengenfelder J, Bryant D, Diamond B, Kalmar J, Moore N, DeLuca J. Processing speed interacts with working memory efficiency in multiple sclerosis. Arch Clin Neuropsychol 2006; 21: 229-38.

(9) Stegen S, Stepanov I, Cookfair D, Schwartz E, Hojnacki D, Weinstock-Guttman B, et al. Validity of the California Verbal Learning Test-II in multiple sclerosis. Clin Neuropsychol 2010; 24: 189202.

(10) Thornton A, Raz N, Tucke K. Memory in multiple sclerosis: contextual encoding deficits. $J$ Internat Neuropsychol Soc 2002; 8: 395-409.

(11) Chiaravalloti N, DeLuca J. Cognitive impairment in multiple sclerosis. The lancet 2008; 7: 1139-51.

(12) Introzzi I, Canet L, Andrés ML, Richard's M. Análisis de procesos de codificación y recuperación en pacientes con Esclerosis Multiple. Revista Chilena de Neuropsicología 2007; 2: 34-43.

(13) Freitas S, Callegaro D, Pompéia S, Orlando F. Working memory impairment in multiple sclerosis relapsing-remitting patients with episodic memory deficits. Arquivos de Neuro-psiquiatria 2010; 68: 205-11.

(14) Grigsby J, Ayarbe S, Kravcisin N, Busenbark. Working memory impairment among persons with chronic progressive multiple sclerosis. Journal of Neurology 1994; 241: 125-31.

(15) Sfagos C, Papageorgiou C, Kosma K, Kodopadelis E, Uzunoglu N, Vassilopoulos, D, et al. A. Working memory deficits in multiple sclerosis: a controlled study with auditory P600 correlates. J Neurol Neurosurg Psychiatry 2003;74: 1231-35.

(16) Sperling RA, Guttmann CR, Hohol MJ, Warfield SK, Jakab M, Parente M, et al. Regional magnetic resonance imaging lesion burden and cognitive function in multiple sclerosis: a longitudinal study. Arch Neurol 2001; 58: 115-21.

(17) Newman SD, Carpenter PA, Varma S, Just MA. Frontal and parietal participation in problem solving in the Tower of London: fMRI and computational modeling of planning and high-level perception. Neuropsychologia 2003; 41(12); 1668-82. 


\section{ORIGINALES Y REVISIONES}

(18) Heesen C, Schulz KH, Fiehler J, Von der Mark U, Otte C, Jung R, et al. Correlates of cognitive dysfunction in multiple sclerosis. Brain, Behavior, and Immunity 2010; 24: 1148-55.

(19) Parente MAMP, Fonseca RP. Substratos neurais da linguagem e seus fatores biológicos e sociais. In: Landeira-Fernandez, J, Araujo Silva, MTA, Editors. Intersecções entre psicologia e neurociências. Medbook; 2007:189-215.

(20) Poser D, Paty D, Scheinberg L, McDonald W, Davis F, Eberg C. (1983). New diagnostic criteria for multiple sclerosis: guiedlines for research protocols. Annals of Neurology 1983; 13: 227-31.

(21) Folstein M, Folstein S, McHugh P. Mini-Mental State: a practical method for grading the cognitive state of patients for the clinician. Journal of Psychiatric Research 1975; 12: 189-98.

(22) Benedet M, Alejandre M. (1998). Test de Aprendizaje Verbal España-Complutense (TAVEC). Madrid: TEA.

(23) Higueras Y, Jiménez-Martín I, Muñoz L, Mateo D, de Andrés C. Características clínicas del deterioro cognitivo en la demencia por esclerosis múltiple: estudio transversal. Revista Española de Esclerosis Múltiple 2009; 1: 5-15.

(24) Martinena P, Blas J, Medina C, Baños I, Sabanés A, Vicens J, et al. Esquizotipia y memoria verbal en población general adolescente. Psicothema 2006; 18: 439-46.

(25) Drew M, Tippet L, Starkey N, Isler R. Executive dysfunction and cognitive impairment in a large community-based sample with Multiple Sclerosis from New Zealand: A descriptive study. Arch Clin Neuropsychol 2008; 23: 1-19.

(26) García-Molina A, Tirapú-Ustárroz J, Luna-Lario P, Ibáñez J, Duque P. ¿ Son lo mismo inteligencia y funciones ejecutivas? Rev Neurol 2010; 50: 738-43.

(27) Lezak M. Neuropsichological Assessment. New York: McGraw Hill. 2002.

(28) Nagy H, Bencsik K, Rajda C, Benedek K, Beniczky S, Keri S, et al. The effects of reward and punishment contingencies on decision-making in multiple sclerosis. J Int Neuropsychol Soc 2006; 12: $559-65$.

(29) Arango J, Fernández S, Ardila A. Las demencias: Aspectos clínicos, neuropsicológicos y tratamiento. (2003). México: Manual

(30) Sartori E, Edan G. Assessment of cognitive dysfunction in multiple sclerosis. J Neurol Sci 2006; 245: 169-75. 